\title{
Association between Neurofibromatosis Type I and Central Giant Cell Lesion: Case Report
}

\author{
Monyque Cunha Trindade ${ }^{1}$, Fernando Melhem Elias ${ }^{2 *}$, Rodrigo Chenu Migliolo, Nathalia Cayuela Nogueira ${ }^{1}$ and Flávio Welington da Silva \\ Ferraz $^{3}$ \\ ${ }^{1}$ Department of Oral and Maxillofacial Surgery at the University Hospital of the University of Sao Paulo, Brazil \\ ${ }^{2}$ School of Dentistry and Department of Oral and Maxillofacial Surgery at the University Hospital of the University of Sao Paulo, Brazil
}

${ }^{3}$ Department of Oral and Maxillofacial Surgery at the University Hospital of the University of Sao Paulo, Brazil

\begin{abstract}
Neurofibromatosis type I (NF1), or Von Recklinghausen disease, is characterized by café-au-lait spots and neurofibromas of the skin, bone defects, iris hamartomas (Lisch nodules) and tumors in the central nervous system. The central giant cell lesion (CGCL) is a non-neoplastic entity, intra-osseous, radiolucent, uni or multilocular, well-defined edges, and it can change the position of the teeth, but rarely causes resorption of them. The association between CGCL and NF1 has been described in the literature. Patient C.R.P, 42 years old, female, who has NF1 attended the service presenting asymptomatic swelling in the anterior maxilla and discrete painless intraoral bulge in the anterior mandible with approximate evolution of one year. Incisional biopsy was performed, and the result was CGCL. The patient didn't have hyperparathyroidism. Treatment consisted of curettage of the lesion and bone regularization performed three years ago, without signs of recurrence. The occurrence of CGCL in multiple locations is uncommon and it is usually associated with hyperparathyroidism, cherubism, and can be also found in syndromes such as: Ramon Jaffe Campanacci, Noonan-like and NF1. The patient presented four features of NF1 (multiple skin neurofibromas, caféau-lait spots, Lisch nodules and freckles in the armpits) and CGCL in two locations, which corroborate the existence of this association in the literature. The apparent relationship between NF1 and an increased incidence of CGCL $s$ in the jaws could represent a coincidental association, genetic relationship, or susceptibility to developing CGCL in abnormal bone quality. In cases of multiple CGCL, the presence of hyperparathyroidism, cherubism and syndromes such as NF1, should be considered. One realizes the importance of investigation of systemic factors and clinical signs for performing differential diagnosis. The differentiation of these lesions in aggressive and nonaggressive should be done to improve individual treatment plan. Surgical curettage is acceptable for the treatment of non-aggressive CGCL.
\end{abstract}

\section{Introduction}

Neurofibromatosis type I (NF1), also known as Von Recklinghausen's neurofibromatosis is a common genetic autosomal dominant hereditary disorder that affects multiple body systems [16]. It is the most common type of neurofibromatosis, accounting for $90 \%$ of all cases. The estimated prevalence is $1: 3000$ births [2,5,6]. Approximately $50 \%$ of cases occur in patients without affected relatives, what is believed to represent spontaneous mutations [4].

In NF1 central and peripheral nervous system are often affected. Bone abnormalities are reported between $50 \%$ to $70 \%$ and oral manifestations in $70 \%$ of cases [2,5]. The most common oral lesions are increased fungiform papillae, intraosseous cystic lesions, branches of the mandibular canal, and enlarged foramen and mandibular canal. Only $25 \%$ of patients with neurofibromatosis present intraoral neurofibromas $[2,4]$.

In addition, specific skeletal injuries of the jaws include coronoid process enlargement, condylar deformity, condylar neck stretching, irregularities in mandibular cortical, thinning and lateral curvature of mandibular ramus $[4,6]$.

There is great variability of phenotypes, with $50 \%$ of patients having only minor manifestations. Café-au-lait spots usually begin to develop before 4 years old. Lisch nodules may appear after 3 years old and armpit freckles can be seen after 7 years old. The latter symptoms are the neurofibromas $[7,8]$.

The vascularization is dense and vascular malformations are seen commonly in NF1. Severe bleeding is usually associated with surgery for plexiform neurofibroma. Enlargement of the mandibular foramen can be explained by the increase of plexiform neurofibroma of the inferior alveolar nerve [6].

The patient must present two of the seven following criteria for establishing the diagnosis of NF1 $[2,4,6]$.
1. Six or more café-au-lait macules over $5 \mathrm{~mm}$ in greatest diameter in prepuberal individuals and over $15 \mathrm{~mm}$ in greatest diameter in postpuberal individuals.

2. Two or more neurofibromas of any type, or one plexiform neurofibroma.

3. Freckling in the axillary or inguinal regions.

4. Optic glioma (optical tumor of glial cells)

5. Two or more Lisch nodules (iris hamartomas)

6. A distinctive osseous lesion:

a. Sphenoid dysplasia

b. Thinning of long bone cortex with or without pseudoartrosis

7. A first degree relative with NF1 by the above criteria.

The tumors most commonly found in patients with NF1 include neurofibromas and optical gliomas. Malignant tumors of peripheral nerve sheath develop from pre-existing plexiform neurofibromas in

*Corresponding author: Fernando Melhem Elias, Associate Professor, Schoo of Dentistry and University Hospital, Director, Residency Program on Oral and Maxillofacial Surgery, University of Sao Paulo - Brazil, Tel: +55-11-98294-2299; E-mail: fmelias@usp.br, fernandomelhem@hotmail.com

Received December 06, 2014; Accepted December 07, 2014; Published January 05, 2015

Citation: Trindade MC, Elias FM, Migliolo RC, Nogueira NC, Ferraz FWDS (2015) Association between Neurofibromatosis Type I and Central Giant Cell Lesion: Case Report. Pigmentary Disorders 2: 161. doi:10.4172/2376-0427.1000161

Copyright: (C) 2015 Trindade MC, et al. This is an open-access article distributed under the terms of the Creative Commons Attribution License, which permits unrestricted use, distribution, and reproduction in any medium, provided the original author and source are credited. 
$2-16 \%$ of patients, and is the leading cause of death in patients with NF1 $[2,4]$

The CGCL is defined as an intraosseous lesion of fibro-cellular tissue containing multiple hemorrhagic foci, aggregations of multinucleated giant cells and occasionally trabecular bone $[1,3,8]$.

It is a non-neoplastic bone lesion, covering around $7 \%$ of all benign tumors of jaws $[1,6]$. It presents as a solitary, radiolucent, multilocular lesion in the mandible or maxilla. Occurs most commonly in young adults, in the first three decades of life, with a predilection for female gender $[1,3,8]$. About two-thirds of lesions affecting the mandible, especially the anterior region and $25 \%$ of these crosses the midline $[2,3]$.

The biological behavior of CGCL ranges from innocent to aggressive, presenting pain, root resorption, paresthesia and relapse after curettage [1,6-8]. No histological or biochemical differences can be found between aggressive or non-aggressive lesions [8].

There is controversy in the literature whether the lesion is a true neoplasm or a reactive response. Several hypotheses have been proposed to explain this pathogenesis, including intraosseous hemorrhage after trauma, association with other pre-existing bone lesion, neoplastic nature, or a reactive idiopathic process $[3,4]$.

CGCL in jaws, especially small lesions, are commonly treated with surgical curettage. Aggressive lesions are occasionally treated by bloc resection. However, this approach can be unsightly. Other treatments for large or multiple lesions include weekly intralesional corticosteroids, subcutaneous or inhaled daily calcitonin and interferon alfa-2 [3].

Recurrences are uncommon with an estimated $75 \%$ success rate after 5 years of conventional surgical therapy. The recurrence rate is higher among boys and young men [4].

Some syndromes have been associated with CGCL, including Noonan syndrome, NF1 and Ramon syndrome. It has been suggested these entities represent different levels of a contiguous gene syndrome, resulting from defects or deletions of two or more genes that are located close to each other in the same region of chromosome [3].

NF1 has overlapping phenotypes with Noonan syndrome in approximately $12 \%$ of patients and multiple giant cell lesions have been reported in patients with Noonan $[2,4]$ syndrome.

Reports of CGCL in patients with NF1 have been described in literature $[2,4,6]$, and the apparent association between NF1 and an increased incidence of CGCL jaws may represent a coincidental association, genetic relationship, or susceptibility to develop CGCL in abnormal bone quality [4]. Multiple CGCL are very rare in the absence of hyperparathyroidism or cherubism [1,4]. The brown tumor of hyperparathyroidism is an osteolytic lesion that results from excessive secretion of parathyroid hormone (PTH). This may occur primarily due to hyperplasia or neoplasia of the parathyroid glands or secondary in association with renal osteodystrophy. Hypercalcemia, hypophosphatemia, and elevated blood levels of PTH characterized hyperparathyroidism [3]. Cherubism is characterized by radiolucency in the quadrants of the jaws. Commonly manifests with progressive and symmetrical enlargement of the mandible and/or maxilla that is noticed between 1 and 4 years old. Dental findings include premature exfoliation of primary teeth. Cervical lymphadenopathy is common. It is probably caused by genetic mutation $[3,5]$.

In 83 cases of CGCL identified two patients had NF1 and one paciente had NF1 and a Noonan-like phenotype. This is at least 3.6\% of all cases CGCL [4]. Alternatively, the association between NF1 and
CGCL could represent a real genetic linkage, resulting from defects or deletions of two or more genes are close together on the same region of chromosome [4].

Edwards et al. believe that the increased incidence of CGCL of the jaws in NF1 patients represents a susceptibility inherent to intraosseous trauma or hemorrhage in previously altered bone for dysplasia. This abnormal bone quality can be more likely to develop CGCL, like lesions in response to yet undefined factors such as excessive mechanical stress or vascular fragility [4].

\section{Case Report}

Patient C.R.P., 42 years old, female, who has NF1 (Figure 1), presenting expansive lesions in the maxilla and mandible with evolution of 1 year (Figures 2 and 3). Physical exam revealed café-au-lait spots (Figure 4), armpits freckles (Figure 5), skin neurofibromas (Figure 6) and Lisch nodules (Figure 7). She presented facial asymmetry with increased left cheek (Figure 1). In oroscopy, there was an expansive lesion in the maxilla, bulging the left maxillary ridge, with purplish coloration, and slight increase in anterior mandible (Figures 2 and 3). Computerized tomography revealed lesions affecting the bone structure of the jaws (Figures 8 and 9).

PTH, alkaline phosphatase, calcium and phosphate blood levels were normal, ruling out the possibility of hyperparathyroidism.

Incisional biopsy of both lesions was performed, and the histopathological result was CGCL. Curettage and marginal ostectomy of both lesions were done, without relapse after 36 months of follow-up.

\section{Discussion}

CGCL are considered reactive lesions that occur most commonly as single lesions ${ }^{2}$. It involve the mandible more frequently than maxilla. It is usually an asymptomatic lesion, or with mild localized pain, causing facial asymmetry. Rarely it involves other facial bones. The teeth are usually vital and without reabsorption [1].

The occurrence of multiple lesions is uncommon and is usually associated with hyperparathyroidism and cherubism [2,6]. Multiple CGCL have been associated with conditions like Ramon, Jaffe Campanacci, Noonan-like syndromes and NF1 [2].

Ruggieri et al. reported that multiple CGCL suggest a possible diagnosis of brown tumor of hyperparathyroidism. And there are four

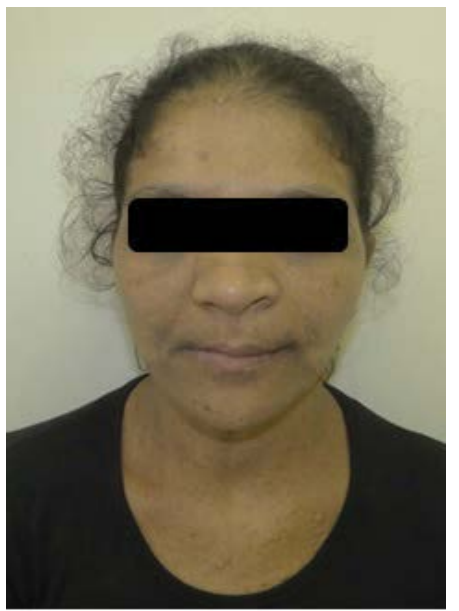

Figure 1: The patient presenting increased left cheek. 
Citation: Trindade MC, Elias FM, Migliolo RC, Nogueira NC, Ferraz FWDS (2015) Association between Neurofibromatosis Type I and Central Giant Cell Lesion: Case Report. Pigmentary Disorders 2: 161. doi:10.4172/2376-0427.1000161

Page 3 of 4

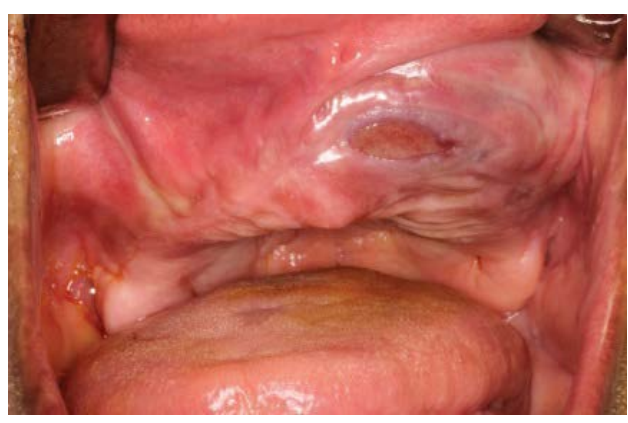

Figure 2: Intraoral lesions aspect 1.

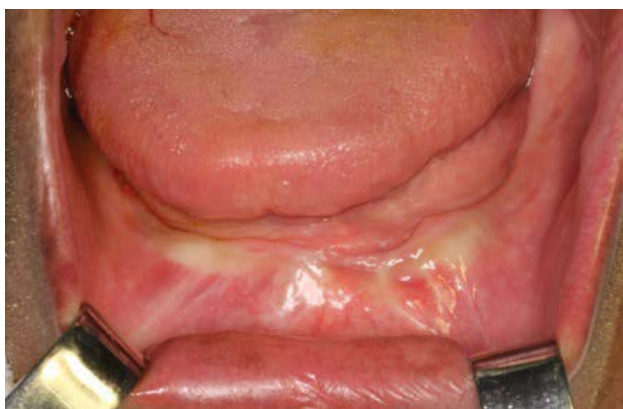

Figure 3: Intraoral lesions aspect 2.

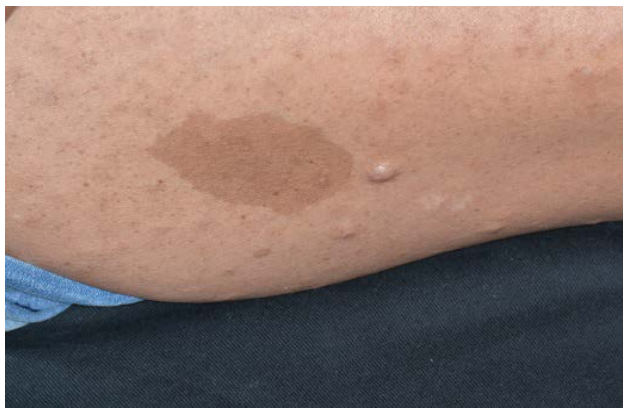

Figure 4: Café-au-lait Spot.

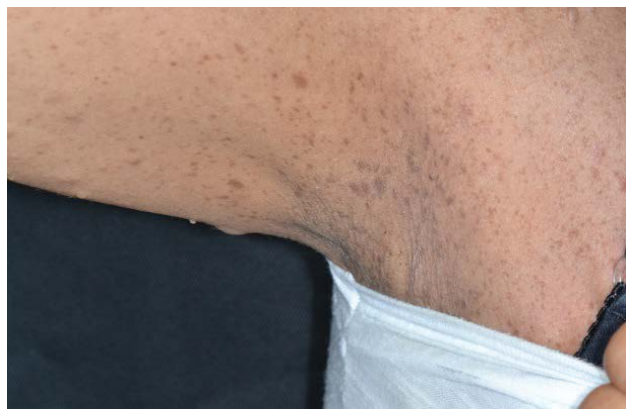

Figure 5: Armpit frekles.

previously reported cases of patients having normal calcium levels with similar lesions to those seen in their patients, in which PTH level were normal [5].

According to Friedrich RE, Mautner VF and Scheuer HA (2007), a skeletal find associated with NF1 is CGCL. This lesion was also found in the jaws of patients with this syndrome. A genetic link between the two disorders is not well established currently [7].
The association of neurofibromatosis and CGCL has been reported in cases with and without hyperparathyroidism. Cherubim has also been associated with NF1 [6]. This could indicate a relationship between NF1 and CGCL [8].

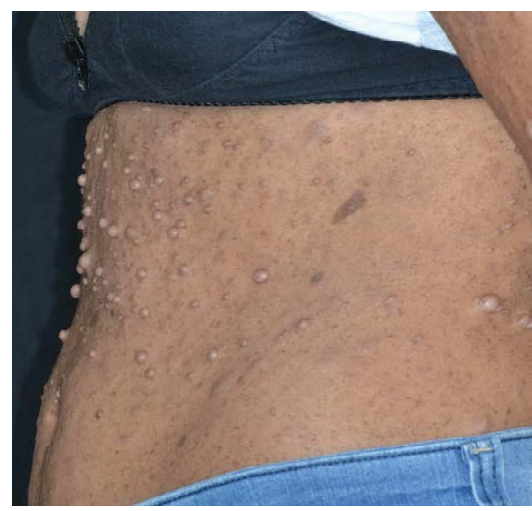

Figure 6: Skin neurofibromas.

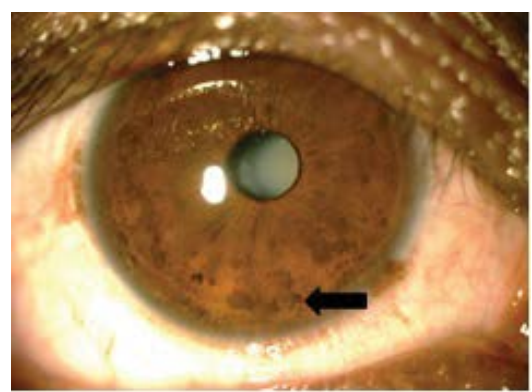

Figure 7: Lisch nodules.

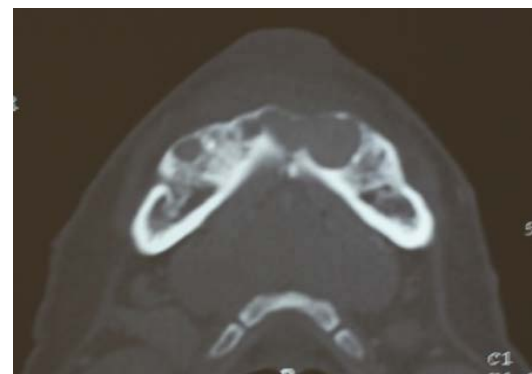

Figure 8: CT scans showing expansive intraosseus lesions.

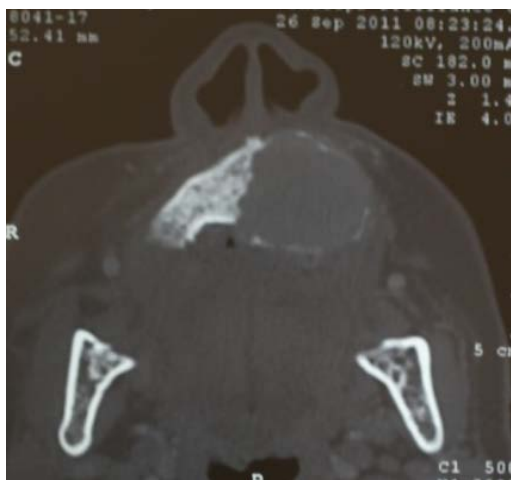

Figure 9: CT scans showing expansive intraosseus lesions 
Smith et al. affirm that the absence of hypercalcemia does not always exclude the hyperparathyroidism and that several factors must be monitored $[2,5]$. In the case reported, the patient had normal values of total calcium, phosphate and alkaline phosphatase [5].

A slight increase in PTH values have been described in the normal population. In this review, neither NF1 nor Noonan syndrome predisposed patients to elevated PTH [2].

Chrcanovic et al. reported that the occurrence of multiple CGCL has been described in association with NF1 and a genetic mutation was found in a family affected by both disorders. Friedrich et al. showed loss of heterozygosity of the NF1 gene in the genetic analysis of CGCL of a seven years old patient, suffering from NF1, using seven microsatellite markers [6].

Regarding the monitoring results, CGCL may recur within 2 years after surgery. Radical surgical approach proved to be effective for aggressive lesions, but this causes inevitable bone loss, dental, dental germs and functional disorders of the inferior alveolar nerve [6].

\section{Conclusion}

Few cases of multiple CGCL of the jaw in patients without hyperparathyroidism have been reported in the literature. Multiple foci of CGCL usually appear in cases of hyperparathyroidism and are known as brown tumors.

For multiple CGCL of the jaw, hyperparathyroidism, cherubism and some syndromes, such as NF1 should be considered in the differential diagnosis.

The possible relationship between CGCL and NF1, which can bring some impact in the treatment of this tumor, still need to be elucidated.
Differentiation between aggressive and nonaggressive CGCL should be considered to improve individual treatment plan. Surgical curetage is acceptable for the treatment of jaw's CGCL without signs of aggression.

\section{References}

1. Ardekian L, Manor R, Peled M, Laufer D (1999) Bilateral central giant cell granulomas in a patient with neurofibromatosis: report of a case and review of the literature. J Oral Maxillofac Surg 57: 869-872.

2. Yazdizadeh M, Tapia JL, Baharvand M, Radfar L (2004) A case of neurofibromatosis-Noonan syndrome with a central giant cell granuloma. Oral Surg Oral Med Oral Pathol Oral Radiol Endod 98: 316-320.

3. Edwards PC (2005) Bilateral central cell granulomas of the mandible in na 8-year-ord girl with Noonan syndrome (Noonan-like/multiple giant cell lesion syndrome). Oral Surg Oral Med Oral Pathol Oral Radiol Endod 99: 334-340.

4. Edwards PC, Fantasia JE, Saini T (2006) Clinically aggressive central giant cell granulomas in two patients with neurofibromatosis . Oral Surg Oral Med Oral Pathol Oral Radiol Endod 102: 765-772.

5. Ruggieri M, Pavone V, Polizzi A (1999) Unusual form of recurrent giant cell granuloma of the mandible and lower extremities in a patient with neurofibromatosis type 1. Oral Surg Oral Med Oral Pathol Oral Radiol Endod 87: 67-72.

6. Chrcanovic BR, Gomez RS, Freire-Maia B (2011) Neurofibromatosis type associated with bilateral central giant cell granuloma of the mandible. Journal of Cranio-Maxillo-Facial Surgery 39: 538-543.

7. Friedrich RE, Mautner VF, Scheuer HA (2007) Loss of heterozygosity in tumor cells of a recurrent mandibular giant cell granuloma in neurofibromatosis type 1. Anticancer Res 27: 2079-2083.

8. Krammer $\mathrm{U}$, Wimmer $\mathrm{K}$, Wiesbauer $\mathrm{P}$, Rasse $\mathrm{M}$, Lang $\mathrm{S}$, et al. (2003) Neurofibromatosis 1: A novel NF1 mutation in an 11-year-old girl with a giant cell granuloma. J Child Neurol 18: 371-373. 